Received: 2021/06/12, Revised: 2021/06/25, Accepted: 2021/06/28, Published: 2021/06/30 (๑2021 Satoshi Hattori et al.; Licence Physical Activity and Nutrition. This is an open access article distributed under the terms of the creative commons attribution license (https:/l creativecommons.org/licenses/by-nc/2.0/), which permits unrestricted use, distribution, and reproduction in any medium, provided the orginal work is properly cited.

${ }^{*}$ Corresponding author : Naomi Omi, Ph.D.

Faculty of Health and Sport Sciences, University of Tsukuba, 1-1-1 Tennodai, Tsukuba, Ibaraki, 305-8574, Japan

Tel: +81-29-853-6319 / Fax: +81-29-853-6507

E-mail: omi.naomi.gn@u.tsukuba.ac.jp

(๑2021 The Korean Society for Exercise Nutrition

\section{Effect of ginger extract ingestion on skeletal muscle glycogen contents and endurance exercise in male rats}

\author{
Satoshi Hattori ${ }^{1, \S} /$ Naomi Omi ${ }^{1, \S, *}$ / Zhou Yang ${ }^{2}$ / \\ Moeka Nakamura ${ }^{2}$ / Masahiro Ikemoto ${ }^{3}$ \\ 1. Faculty of Health and Sport Sciences, University of Tsukuba, Tsukuba, Japan \\ 2. Comprehensive Human Sciences, University of Tsukuba, Tsukuba, Japan \\ 3. Ikeda Food Research Co., Ltd, Fukuyama, Hiroshima, Japan \\ $\S$. These authors contributed equally to this work
}

\section{INTRODUCTION}

[Purpose] Skeletal muscle glycogen is a determinant of endurance capacity for some athletes. Ginger is well known to possess nutritional effects, such as anti-diabetic effects. We hypothesized that ginger extract (GE) ingestion increases skeletal muscle glycogen by enhancing fat oxidation. Thus, we investigated the effect of GE ingestion on exercise capacity, skeletal muscle glycogen, and certain blood metabolites in exercised rats.

[Methods] First, we evaluated the influence of GE ingestion on body weight and elevation of exercise performance in rats fed with different volumes of GE. Next, we measured the skeletal muscle glycogen content and free fatty acid (FFA) levels in GE-fed rats. Finally, we demonstrated that GE ingestion contributes to endurance capacity during intermittent exercise to exhaustion.

[Results] We confirmed that GE ingestion increased exercise performance $(p<0.05)$ and elevated the skeletal muscle glycogen content compared to the nonGE-fed (CE, control exercise) group before exercise (Soleus: $p<0.01$, Plantaris: $p<0.01$, Gastrocnemius: $p<0.05)$. Blood FFA levels in the GE group were significantly higher than those in the CE group after exercise $(p<0.05)$. Moreover, we demonstrated that exercise capacity was maintained in the CE group during intermittent exercise $(p<0.05)$.

[Conclusion] These findings indicate that GE ingestion increases skeletal muscle glycogen content and exercise performance through the upregulation of fat oxidation

[Key words] ginger extract, glycogen, exercise, FFA, blood glucose, blood lactate
Skeletal muscle glycogen content changes dynamically due to external stimuli, such as exercise and nutrition. Studies in the 1960s clarified that the restriction of glycogen storage leads to an inability to continue exercise $^{1}$, whereas glycogen depletion provides a strong driving force for its supercompensation (i.e., glycogen loading) and causes significant improvements in exercise performance ${ }^{2}$. In addition, skeletal muscle glycogen status plays a vital role in the regulation of cellular adaptation via multiple signal pathways from exercise stimuli ${ }^{3}$. Thus, dietary strategies to maximize muscle glycogen synthesis are valid for enhancing the performance during important workouts or competitive events.

Glycogen storage in the skeletal muscle has various metabolic impacts, especially for the mobilization of energy substrates during endurance exercise ${ }^{4}$. It is well known that the ergogenic effect of carbohydrate (CHO) loading also enhances skeletal muscle glycogen utilization during prolonged exercise ${ }^{5,6}$; therefore, nutritional treatment is ideal for increasing glycogen levels without using $\mathrm{CHO}$ substrates more than necessary. Thus, a nutritional strategy is required to up-regulate skeletal muscle glycogen and maintain compatible $\mathrm{CHO}$ mobilization for competitive sports.

The co-ingestion of CHO-rich foods with other macronutrients or agents (e.g., protein, creatine, and caffeine supplementation) results in a further acceleration of muscle glycogen synthesis ${ }^{4}$. However, although these methods increase skeletal muscle glycogen storage, they also elevate the $\mathrm{CHO}$ consumption rate. Thus, we present another solution that up-regulates glycogen levels and inhibits $\mathrm{CHO}$ utilization by enhancing fat oxidation in skeletal muscle. Ginger (Zingiber officinale Roscoe) is widely consumed and is known to have anti-diabetic, anti-inflammatory, and anti-oxidant effects ${ }^{7}$. Moreover, ginger extract (GE) has various impacts in determining enzymes that control the use of energy substrates. This extract up-regulates fat oxidation via the peroxisome proliferator-activated receptor delta (PPAR $\delta$ ) signaling, thereby attenuating high-fat diet-induced obesity in mice ${ }^{8}$. These findings suggest that GE contributes to the maintenance of skeletal muscle glycogen by enhancing fat metabo- 
lism during endurance exercise.

GE may enhance endurance capacity through energy metabolism, although this effect is not well known. Therefore, in the present study, we examined the influence of GE ingestion on endurance performance and metabolite levels in rats during endurance exercise.

\section{METHODS}

\section{Experimental strategy}

We outlined the following experimental strategy to investigate the effect of GE ingestion on skeletal muscle glycogen content and endurance exercise. First, we confirmed whether the extracts increased the exercise capacity of GEfed rats. Second, we evaluated the effect of GE on blood metabolites and skeletal muscle glycogen content in rats to estimate the influence of GE on energy metabolism. Third, we tested the efficacy of GE ingestion on exercise capacity across the recovery periods. Male Sprague Dawley (SD) rats were purchased from CLEA Experimental Animals Supply (CLEA Japan, Inc., Tokyo, Japan) and cared for according to the "Guiding Principles for the Care and Use of Animals." The rats were fed a standard diet CE-2 (CLEA Japan, Inc., Tokyo, Japan). The room temperature was maintained at $25 \pm 1{ }^{\circ} \mathrm{C}$, and humidity was maintained under a constant 12:12-hour light/dark cycle (light: 8:00 a.m.-8:00 p.m.). Distilled water was freely available for drinking ad libitum. The animal care and experimental procedures of this study were approved by the Experimental Animal Committee of the University of Tsukuba.

\section{Dietary food}

Laboratory animals in the control group (without GE) were fed CE-2 (CLEA Japan, Inc. Tokyo, Japan), and for GE-fed rats, CE-2 was mixed ginger extract powder (Ikeda Food Research Co., Ltd, Hiroshima, Japan; Table 1).

\section{Experiment 1: Effect of GE ingestion on exercise ca- pacity of rats}

SD rats ( $\mathrm{n}=28,6$ weeks old) were subjected to 25 days of exercise training after a habitation period of 3 days, with ad libitum feeding. After these durations, the animals were randomly divided into three groups (CE: control exercise group; [n=7], LGE: low ginger exercise group; [n=11], HGE: high ginger exercise group; $[\mathrm{n}=10]$ ) as their body weight was the same. The rats were administered GE after exercise training, and were therefore subjected to an all-out test.

Experiment 2: Effect of GE ingestion on blood metabolites and skeletal muscle glycogen content of rats

SD rats ( $\mathrm{n}=20,6$ weeks old) were divided into two groups (CE: control exercise group; $[\mathrm{n}=10]$ and GE: ginger exercise group; $[\mathrm{n}=10]$ ) after 3 days of habitation and 7 days of exercise training. These rats were administered GE after exercise training, and they were tested with an all-out test similar to that in Experiment 1. Blood samples were collected before, immediately after (post1), and $1 \mathrm{~h}$ after exercise (post2).

\section{Experiment 3: Efficacy of GE ingestion for maintain-} ing exercise capacity across recovery periods

This experiment was conducted based on Experiment 2. SD rats $(\mathrm{n}=20,6$ weeks old) were also divided into two groups (CE: control exercise group; [n=10] and GE: ginger exercise group; [n=10]) after 3 days of habitation and 7 days of exercise training. They were fed GE after exercise training and made to attempt an all-out test. Furthermore, these rats underwent the same all-out test after a 1 hour recovery period to evaluate the influence of GE ingestion on the recovery period.

\section{Exercise habitation and training}

The rats were exercised on a treadmill (Natsume Seisakusho Co, Ltd, Tokyo, Japan) to habituate them to exercise conditions $(10 \mathrm{~m} / \mathrm{min}, 15 \mathrm{~min}$ and $25 \mathrm{~m} / \mathrm{min}, 30 \mathrm{~min})$, and they were trained on the same experimental equipment $(25 \mathrm{~m} / \mathrm{min}, 30 \mathrm{~min})$ for 25 days. Warming up and cooling down were performed before and after exercise training (15 $\mathrm{m} / \mathrm{min}, 2 \mathrm{~min}$ ).

\section{All-out test}

This test was carried out $24 \mathrm{~h}$ after the final exercise training. The animals were exercised at $30 \mathrm{~m} / \mathrm{min}$ on a treadmill until they could not maintain this exercise intensity. The animals were exercised for $110 \mathrm{~min}$ and the treadmill speed was then accelerated by $2 \mathrm{~m} / \mathrm{min}$ until all-out. All-out was declared when the rats could not keep up with the exercise speeds, and they could not restore their bodies from the supine position.

\section{Skeletal muscle and liver extraction}

Skeletal muscle of the left leg (soleus, plantaris, gastrocnemius muscle) and liver were extracted under anesthesia. The samples were frozen in liquid nitrogen after trimming for storage.

\section{Measurement of skeletal muscle and liver glycogen contents}

The glycogen concentration of skeletal muscles and liver was measured by the amyloglucosidase method ${ }^{9}$. Briefly, these tissues were homogenized in perchloric acid below freezing using dry ice to inhibit the breakdown of metabolites (Power Homogenizer Portable S-203, AS ONE Corporation, Osaka, Japan). After homogenization, ultrapure water was added and the tissues were re-homogenized. The lysates were separated by centrifugation $\left(250 \mathrm{rpm}, 15 \mathrm{~min}, 4^{\circ} \mathrm{C}\right)$ and the supernatants were stored at $-80^{\circ} \mathrm{C}$. These samples were added to $50 \mathrm{mM} \mathrm{NaAC}$ buffer and rotary incubated at $120 \mathrm{rpm}$ for $60 \mathrm{~min}$ at $37{ }^{\circ} \mathrm{C}$ to disassemble glycogen. Glucose reagent was added to these samples and incubated for $20 \mathrm{~min}$ at room temperature. Glycogen concentrations were analyzed using a fluoro-spectrophotometer (FP-8300, JASCO Co., Tokyo, Japan). 
Evaluation of blood glucose, free fatty acid (FFA), and lactate levels

Blood samples were collected from the tail vein before and after the all-out test. Blood glucose levels were evaluated using glucose measurements (Onetouch UltravueTM, Johnson \& Johnson, Tokyo, Japan). FFA levels were evaluated using a spectrophotometer (V-630BIO, JASCO Corporation, Tokyo, Japan) by measuring the enzymatic reactions (acyl-CoA synthase, acyl-CoA oxidase, and peroxidase) ${ }^{10}$. Blood lactate levels were estimated through lactate measurements (Lactate $\mathrm{Pro}^{\mathrm{TM}}$ 2, ARKRAY, Inc., Kyoto, Japan).

\section{Statistical Analysis}

All data are expressed as mean \pm standard error (SE) and were analyzed using SPSS version 25 (IBM Japan, Tokyo, Japan). One-way analysis of variance was used to test for statistically significant differences among the groups. If a significant difference was detected among groups, the groups were further evaluated using the post-hoc Scheffe test. The significance level for major effects was set at $\mathrm{p}<0.05$.

\section{Results}

Experiment 1: Effect of GE ingestion on endurance capacity of rats.

There were no significant differences in food intake and final body weight among the groups (Supplementary Figure 1A, B), although differences in body weight significantly decreased depending on the volume of ingested GE in these groups (Supplementary Figure 1C, CE vs. HGE: $\mathrm{p}<0.05$ ). Similarly, the running distance significantly increased with the volume of ingested GE in these groups (Sup Figure 1D, CE vs. HGE: $\mathrm{p}<0.05$ ).

Experiment 2: Effect of GE on blood metabolites and skeletal muscle glycogen content before and after endurance exercise.

The final body weight, body weight gain, and food efficiency significantly decreased in the GE group compared to the CE group (Table $1, p<0.001, p<0.001$, and $p<0.05$, respectively). Blood glucose levels before exercise were significantly lower in the GE group than in the $\mathrm{CE}$ group (Figure 1A, $\mathrm{p}<0.05$ ). However, the blood glucose levels significantly increased post-exercise in both groups compared to the pre-exercise levels (Figure 1A, CE group: $p<0.001$ and GE group: $\mathrm{p}<0.01$ ). FFA levels in the GE group were significantly higher than those in the CE group after exer-

Table 1. Final body weight, body weight gain, food intake, and food efficiency after GE ingestion. Values are represented as mean $\pm \mathrm{SE}$.

\begin{tabular}{|ccc} 
& CE & GE \\
\hline Final body weight $(\mathrm{g})$ & $312.8 \pm 2.6$ & $285.6 \pm 4.7^{* * *}$ \\
\hline Body weight gain $(\mathrm{g})$ & $14.8 \pm 2.0$ & $-7.8 \pm 3.7^{* * *}$ \\
Food efficiency $(\mathrm{g})^{\mathrm{a})}$ & $0.5 \pm 0.1$ & $-1.2 \pm 0.6^{*}$ \\
\hline
\end{tabular}

${ }^{a)}$ Food efficiency was calculated by "body weight gain/food intake." ${ }^{*} p<0.05,{ }^{*} p<0.001$ vs GE group.

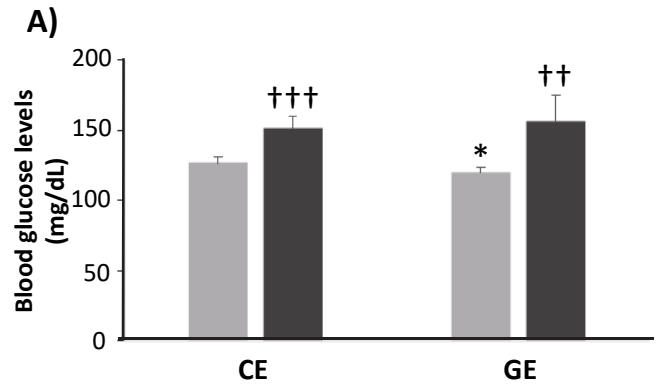

B)
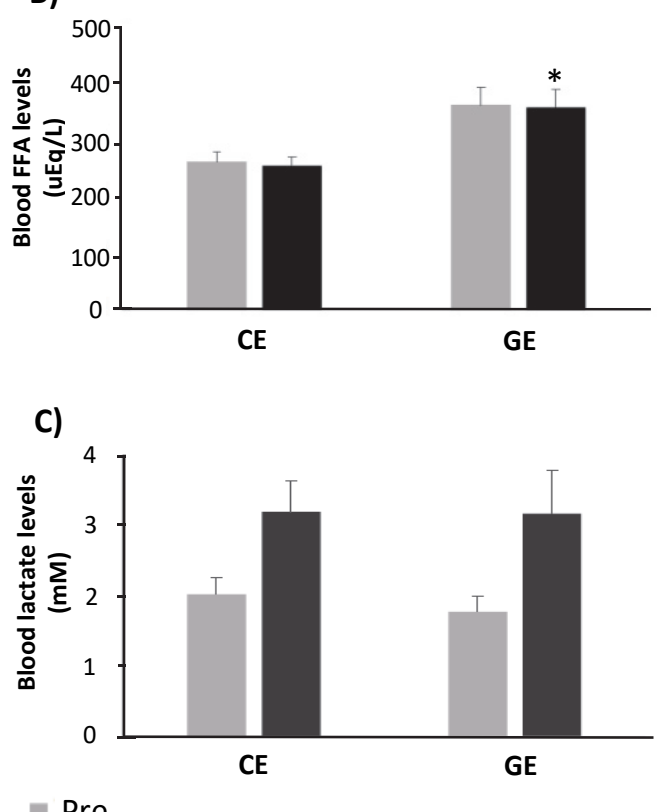

Pre

- Post

Figure 1. Blood glucose (A), free fatty acid (B), and lactate (C) levels of rats. Experimental animals were randomly divided into two experimental groups at the age of 6 weeks (CE: Control exercise group, [ $n=10]$, GE: ginger exercise group [ $n=10])$. Blood samples were collected from the tail vein before and after the all-out test. ${ }^{*} p<0.05$ vs CE group; ${ }^{+1} p<0.01,{ }^{+t} p<0.001$ vs pre. Values are represented as mean $\pm \mathrm{SE}$.

cise (Figure 1B, $\mathrm{p}<0.05)$. There were no differences in blood lactate levels between the groups (Figure 1C).

The skeletal muscle glycogen content in the GE group was significantly higher than that in the $\mathrm{CE}$ group before exercise (Figure $2 \mathrm{~A}-\mathrm{C}$, Soleus: $\mathrm{p}<0.01$, Plantaris: $\mathrm{p}<0.01$, Gastrocnemius: $\mathrm{p}<0.05$ ). The skeletal muscle glycogen content in the GE group was significantly higher than that in the CE group post-exercise (Figure $2 \mathrm{~A}-\mathrm{C}$, Plantaris: $\mathrm{p}<0.01$, Gastrocnemius: $\mathrm{p}<0.05$ ). However, there were no differences in liver glycogen content between these groups (Figure 2D).

Experiment 3: Effect of GE ingestion on exercise capacity across recovery periods.

The running distance was significantly higher in the GE group than that in the CE group in the all-out test (Supplementary Figure $2 \mathrm{~A}, \mathrm{p}<0.05$ ). Blood glucose levels in the GE group were significantly lower than those in the $\mathrm{CE}$ 
A)

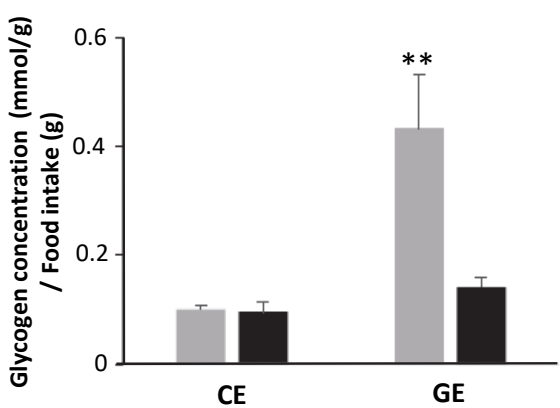

C)

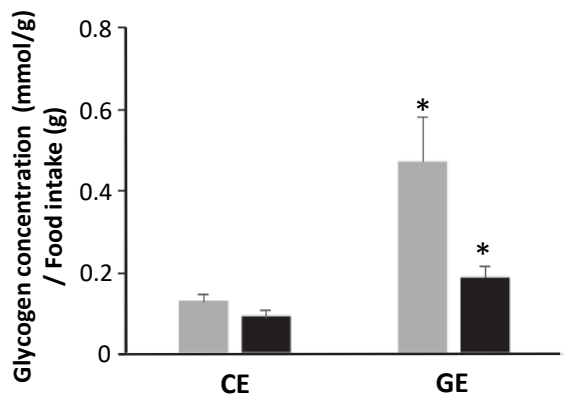

- Pre

- Post
B)

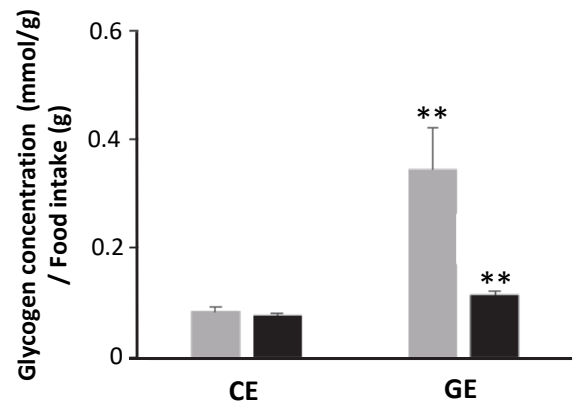

D)

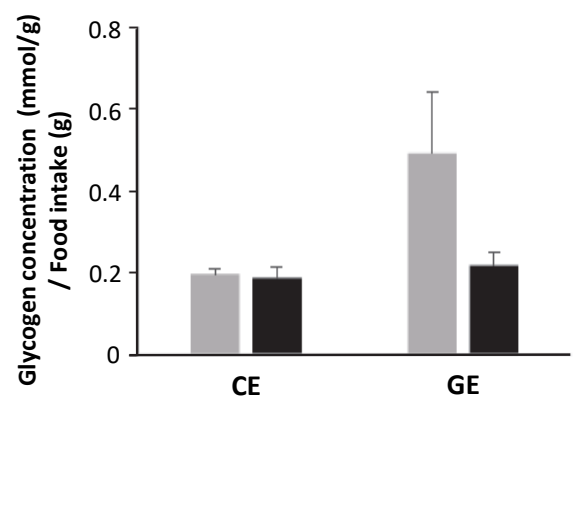

Figure 2. Glycogen concentration in soleus (A), plantaris (B), gastrocnemius (C), and liver (D) per food intake. Experimental animals were randomly divided into two experimental groups at the age of 6 weeks (CE: Control exercise group [ $n=10]$, GE: ginger exercise group [ $n=10]$ ). Skeletal muscles were collected before and after the all-out test. ${ }^{*} p<0.05,{ }^{* *} p<0.01$ vs CE group. Values are represented as mean \pm SE.

group at pre-exercise and post1 stages (Sup Figure 2B, both points: $\mathrm{p}<0.05$ ). The blood glucose levels in the CE group at the post1 stage were significantly higher than those at the pre-exercise stage (Sup Figure 2B, $\mathrm{p}<0.05$ ).

\section{DISCUSSION}

Skeletal muscle glycogen content is a determinant of endurance capacity as an energy substrate and intracellular signaling molecule ${ }^{3}$; therefore, many athletes try to promote rapid and effective restoration of skeletal glycogen using various methods ${ }^{11}$. Some nutritional agents can enhance the rate of muscle glycogen synthesis or enhance glycogen content by providing dietary $\mathrm{CHO}$. However, these methods cannot control the elevation in $\mathrm{CHO}$ oxidation according to the glycogen uptake. Thus, we focused on GE, which is a known nutritional product that increases fat oxidation in skeletal muscle ${ }^{8}$. We hypothesized that GE contributes to exercise performance by up-regulating skeletal muscle glycogen and increasing fat oxidation in animal models. The results showed that GE ingestion elevates running distance, skeletal muscle glycogen content, and blood FFA levels during exercise. These findings indicate that GE ingestion increases exercise performance by mobilizing fatty substrates and avoiding $\mathrm{CHO}$ utilization.

A recent study reported that GE ingestion leads to a re- duction in body weight and enhancement of fat oxidation in obese mice ${ }^{8}$. We found a similar phenomenon for body weight in the GE group in this study (Supplementary Figure 1B). If the results obtained had been affected by changes in energy metabolism in the skeletal muscle, the endurance capacity might have increased depending on the GE intake volume. Notably, the running distance of the GE group was significantly higher than that of the CE group (Sup Figure 1D). These findings indicate that GE ingestion causes a decrease in body weight and an increase in endurance performance.

Exercise has a substantial impact on energy metabolism as various substrates need to be mobilized for meeting the energy demand for contractions of the skeletal muscle. In general, skeletal muscle mobilizes energy substrates via the adenosine triphosphate production process, thereby releasing blood glucose through glycolysis to provide $\mathrm{CHO}$ substrate for the skeletal muscle ${ }^{12}$. In our study, the blood glucose levels significantly increased in both groups after exercise (Figure 1A). However, the FFA levels were higher in the GE group than in the CE group, especially after exercise (Figure 1B). The fuel selection for skeletal muscle contractions is strongly related to endurance capacity; for instance, animals with a high running capacity preferentially mobilize fat substrates compared with those with low running capacity ${ }^{13}$. Thus, these results indicate that GE ingestion enhances endurance performance by up-regulating fat 
substrate utilization during exercise.

Skeletal muscle glycogen content is a determinant of endurance capacity in cellular adaptation and exercise performance ${ }^{14}$. Our study demonstrates that GE ingestion up-regulates skeletal muscle glycogen levels corrected with food intake (Figure 2A-C). Considering the results of FFA levels and skeletal muscle glycogen contents, it can be interpreted that GE ingestion induced elevated fat oxidation and reduced $\mathrm{CHO}$ utilization. Conversely, previous studies have reported that glycogen supercompensation regulates glycogen levels ${ }^{4}$. These methods accelerate CHO consumption rather than high glycogen storage during exercise. Therefore, our study is valuable as an option for promoting fat oxidation to enhance skeletal muscle glycogen content is provided by GE ingestion.

The rapid restoration of muscle glycogen is required between two events or training sessions (e.g., runner's twice-daily workouts). These results indicate that GE ingestion contributes to the enhancement of skeletal muscle glycogen levels. These effects of GE ingestion might be useful for maintaining endurance capacity in the recovery phase during intermittent exercise, such as during athletic competitions. Therefore, we examined whether exercise performance increased because of GE ingestion during two all-out trials. The running distance and blood glucose levels were significantly different between the two groups (Supplementary Figure 2A, B). These findings indicate that GE ingestion contributes to endurance recovery between intermittent exercise periods.

We focused on the possibility that GE ingestion enhanced endurance capacity due to the upregulation of fat oxidation. However, it is not clear if the upregulation pathway actually activated as a molecular biological approach was not employed in the present study. A previous study reported that GE enhances fatty acid utilization via the PPAR $\delta$ pathway in an in vitro model ${ }^{8}$. The results of our study might also be related to a similar mechanism, but further experimentation is necessary to elucidate the molecular mechanism of energy metabolism.

In conclusion, we demonstrated that GE ingestion decreased body weight and enhanced exercise capacity in experimental animals. The cause of this phenomenon may be related to an increase in fat oxidation and skeletal muscle glycogen content. These findings indicate that GE ingestion is useful for improving exercise performance for athletics.

\section{REFERENCES}

1. Hawley JA, Maughan RJ, Hargreaves M. Exercise metabolism: historical perspective. Cell Metab. 2015;22:12-7.

2. Karlsson J, Saltin B. Diet, muscle glycogen, and endurance performance. J Appl Physiol. 1971;31:203-6.

3. Burke LM, Hawley JA. Swifter, higher, stronger: what's on the menu? Science. 2018;362:781-7.

4. Burke LM, van Loon LJC, Hawley JA. Postexercise muscle glycogen resynthesis in humans. J Appl Physiol. 2017;122:1055-67.

5. Bosch AN, Dennis SC, Noakes TD. Influence of carbohydrate loading on fuel substrate turnover and oxidation during prolonged exercise. J Appl Physiol. 1993;74:1921-7.

6. Richter EA, Galbo H. High glycogen levels enhance glycogen breakdown in isolated contracting skeletal muscle. J Appl Physiol. 1986;61:827-31.

7. Ali BH, Blunden G, Tanira MO, Nemmar A. Some phytochemical, pharmacological and toxicological properties of ginger (Zingiber officinale Roscoe): a review of recent research. Food Chem Toxicol. 2008;46:409-20.

8. Misawa K, Hashizume K, Yamamoto M, Minegishi Y, Hase T, Shimotoyodome A. Ginger extract prevents high-fat diet-induced obesity in mice via activation of the peroxisome proliferator-activated receptor $\delta$ pathway. J Nutr Biochem. 2015;26:1058-67.

9. Brodal BP, Gehrken BB. Enzymatic microanalysis of glycogen. Scand J Clin Lab Invest. 1986;46:193-5.

10. Shimizu S, Yasui K, Tani Y, Yamada H. Acyl-CoA oxidase from candida tropicalis. Biochem Biophys Res Commun. 1979;91:10813.

11. Burke LM, Castell LM, Casa DJ, Close GL, Costa RJS, Desbrow B, Halson SL, Lis DM, Melin AK, Peeling P, Saunders PU, Slater GJ, Sygo J, Witard OC, Bermon S, Stellingwerff T. International association of athletics federations consensus statement 2019: nutrition for athletics. Int J Sport Nutr Exerc Metab. 2019;29:73-84.

12. Egan B, Zierath JR. Exercise metabolism and the molecular regulation of skeletal muscle adaptation. Cell Metab. 2013;17:162-84.

13. Overmyer KA, Evans CR, Qi NR, Minogue CE, Carson JJ, Chermside-Scabbo CJ, Koch LG, Britton SL, Pagliarini DJ, Coon JJ, Burant CF. Maximal oxidative capacity during exercise is associated with skeletal muscle fuel selection and dynamic changes in mitochondrial protein acetylation. Cell Metab. 2015;21:468-78.

14. Hawley JA, Lundby C, Cotter JD, Burke LM. Maximizing cellular adaptation to endurance exercise in skeletal muscle. Cell Metab. 2018;27:962-76. 